\title{
Editorial
}

\section{The physics of left ventricular filling: exploring the seemingly obvious}

The assessment of left ventricular diastolic function by its filling characteristics has proved to be a fascinating, if elusive, "holy grail". A complex interaction of factors contributes to the filling velocities at the mitral level and hence makes clinically meaningful interpretation of transmitral velocity very difficult.

Two studies that have recently appeared in the British Heart fournal lend further insight into the complex physiology of transmitral flow. Wang and Gibson added to our knowledge by identifying nine patients in sinus rhythm who lacked both a transmitral Doppler A wave and $M$ mode evidence of mitral annulus motion after the electrocardiographic $\mathbf{P}$ wave. ${ }^{1}$ These nine and a tenth in DDD pacing mode made up $0.2 \%$ of the case load of Wang and Gibson's echocardiography laboratory and thus this is a rare finding. Furthermore, all of these patients had easily recognisable heart disease: five had dilated cardiomyopathy, three had left ventricular hypertrophy, and two had had surgery involving the left atrium. Other conditions in which left atrial standstill has been reported are amyloidosis $^{2}$ and recent electrical cardioversion. Interestingly, nine of the 10 patients in Wang and Gibson's study still had right atrial mechanical activity.

What are the consequences of these findings? The prime concern in atrial fibrillation or standstill is thromboembolism. However, we have learned that left atrial thrombus formation most frequently occurs in the appendage and therefore depends critically on appendage mechanics. Transesophageal pulsed Doppler assessment of inflow and outflow velocities in the left atrial appendage shows mechanical competence even in many patients with atrial fibrillation; none the less, velocities $<25 \mathrm{~cm} / \mathrm{s}$ significantly increase the risk of thromboembolism. ${ }^{3}$ Unfortunately, we do not know whether there was mechanical activity in the appendage in these patients, nor were they investigated for thrombus by transesophageal echocardiography. The relevance of left atrial mechanical standstill in sinus rhythm to thrombus formation in these patients therefore remains uncertain.

Wang and Gibson argue that left atrial standstill might mimic the "restrictive" transmitral Doppler pattern with high $\mathrm{E}$ and absent $\mathrm{A}$ waves found in the presence of very high end diastolic left ventricular stiffness. Therefore, in this condition they prefer to use apexcardiography rather than transmitral Doppler recordings for the diagnosis of atrial standstill. However, as they discuss, restrictive physiology would be readily detectable by increased retrograde pulmonary venous flow during atrial contraction. Thus, if the transmitral Doppler A wave is absent in the presence of sinus rhythm, a transesophageal echocardiogram is helpful to search for thrombus, to assess mechanical activity of the appendage, and to record the pulmonary venous inflow profile by pulsed Doppler. In the future, a new imaging method, Doppler tissue imaging, that can directly measure tissue velocities may aid in the assessment of left atrial mechanics.

Given the load dependency of transmitral flow indices of diastolic left ventricular function, recently several inves- tigators have analysed the hydrodynamics of left ventricular inflow within the left ventricle by both colour Doppler mapping and multigated pulsed Doppler. ${ }^{45}$ They were able to show that in the presence of relaxation abnormalities, the velocity with which inflow reaches the ventricular apex is decreased; this velocity of flow propagation is different from, but not unrelated to, the inflow velocity at the mitral level. At the mitral level both are identical, but flow propagation velocity decreases along its path to the apex, owing to dissipation of kinetic energy.

Fujimoto et al used this concept in an ingenious study attempting to calculate two hydrodynamic variables: (a) early diastolic atrioventricular pressure difference from the length of the accelerated blood column and blood acceleration, and $(b)$ to measure effective orifice area of the nonrestrictive mitral valve. ${ }^{6}$ They found that in dilated cardiomyopathy, the reconstructed early diastolic atrioventricular (and intraventricular) pressure drops are higher and the duration of the inflow "pulse" is shorter than in healthy controls. Although not stated explicitly, Fujimoto et al seem to attribute the higher atrioventricular pressure drop in dilated cardiomyopathy to higher left atrial pressures rather than to stronger ventricular suction or "restoring forces", which have been described by direct pressure recordings from the left ventricle. ${ }^{78}$ They also measured the effective orifice size of the transmitral inflow jet by colour Doppler and by dividing the stroke volume by the time velocity integral. They found a narrowed effective orifice area in dilated cardiomyopathy and explain this as the result of a diminished diastolic increase in the transverse diameter of the ventricle. In summary, Fujimoto et al hypothesise that because of left ventricular diastolic impairment, the hydrodynamics of left ventricular inflow in dilated cardiomyopathy resemble mitral stenosis in that a high early pressure drop and a small effective orifice area are present in comparison with patients without heart disease.

There are some problems with this method. First, velocity at the mitral orifice level is not zero, nor is it elsewhere in the jet. This is of particular concern in dilated cardiomyopathy, where velocities presumably are high owing to increased left atrial pressure at mitral valve opening. If indeed the effective orifice is small in dilated cardiomyopathy, it is surprising that Fujimoto et al did not include the convective term of the Bernoulli equation $\left(4\left(v_{2}^{2}-v_{1}^{2}\right)\right)$ in their calculations, as is usual when a pressure drop across a restrictive orifice is calculated. A finite convective acceleration term would even increase the "reconstructed" pressure drop in dilated cardiomyopathy, underscoring that the atrioventricular gradient at mitral valve opening is high in this condition compared with that in healthy controls. Second, no heart rates are given. Presumably, patients with dilated cardiomyopathy had higher heart rates than the controls. A further question concerns the effects of colour Doppler settings, especially the wall filter, when mitral inflow diameter is measured by this method.

Nevertheless, Fujimoto et al's study furnishes insights 
into the physics of left ventricular filling. The finding of a small effective mitral orifice area despite a large annulus and ventricle in dilated cardiomyopathy together with relatively high initial atrioventricular gradients accords with the steep upslope of the transmitral $\mathrm{E}$ wave seen in severe dilated cardiomyopathy.

A correlation between orifice diameter and mass accelerated at valve opening was demonstrated in an in vitro study ${ }^{9}$; it was also shown that a low accelerated mass and a high initial pressure drop both contribute to a steep $\mathrm{E}$ wave upslope. It is important, though, to remember that high velocities, and thus, a high atrioventricular pressure drop, are present only for a very short time in severe dilated cardiomyopathy; this is in contrast to the situation in mitral stenosis where a fixed orifice causes prolonged high velocity inflow. It would be interesting to see if, and by how much, the initially narrow colour Doppler inflow jet widens during diastole.

This study encourages us to pay attention to diastolic function in diseases in general viewed as diseases of systolic function, such as dilated cardiomyopathy. These diseases frequently entail diastolic dysfunction as well, and thus, therapeutically, correction of both should be sought. Unfortunately, this is no easier for diastolic function than for systolic function.
FRANK A FLACHSKAMPF PETER HANRATH

1 Medizinische Klinik, RWTH Aachen,

Pauwelsstrasse 30, D-52057 Aachen, Germany

1 Wang K, Gibson DG. Non-invasive detection of left atrial mechanical failure in patients with left ventricular disease. $\mathrm{Br}$ Heart $\mathcal{F}$ 1995;74:536-40.

2 Dubrey S, Pollak A, Skinner M, Falk RH. Atrial thrombi occurring during sinus rhythm in cardiac amyloidosis: evidence for atrial electromechanical dissociation. Br Heart $\mathcal{F}$ 1995; 74:541-5.

3 Mügge A, Kühn H, Nikutta P, Grote J, Lopez AG, Daniel WG Assessment of left atrial appendage function by biplane transesophagea echocardiography in patients with nonrheumatic atrial fibrillation: Identification of a subgroup of patients at increased embolic risk. $\mathcal{F} \mathrm{Am}$ Coll Cardiol 1994;23:599-607.

4 Brun P, Tribouilloy C, Duval AM, Iserin L, Meguira A, Pelle G, DoboisRande $\mathrm{I}$. Left ventricular flow propagation during early filling is related to wall relaxation: A color M-mode Doppler analysis. $\mathcal{F} \mathrm{Am}$ Coll Cardio 1992;20:420-32.

5 Stugaard M, Smiseth OA, Risöe C, Ihlen $\mathrm{H}$. Intraventricular early diastolic filling during acute myocardial ischemia. Assessment by multigated color M-mode Doppler echocardiography. Circulation 1993;88:2705-13.

6 Fujimoto S, Parker KH, Xiao HB, Inge KSK, Gibson DG. Early diastolic left ventricular inflow pressures in normal subjects and patients with dilated cardiomyopathy. Reconstruction from pulsed Doppler echocardilated cardiomyopathy. Reconstruction

7 Courtois M, Kovács SJ, Ludbrook PA. Transmitral pressure-flow velocity relation. Importance of regional pressure gradients in the left ventricle during diastole. Circulation 1988;78:661-71.

8 Nicolic S, Yellin EL, Tamura K, Vetter H, Tamura T, Meisner JS, Frater RWM. Passive properties of canine left ventricle: Diastolic stiffness and restoring forces. Circ Res 1988;62:1210-22.

9 Flachskampf FA, Rodriguez L, Chen C, Guerrero L, Weyman AE Thomas JD. Analysis of mitral inertance: a factor critical for early transmitral filling. $f$ Am Soc Echocardiogr 1993;6:422-32. 